\title{
FEM-based Printhead Intelligent Adjusting Method for Printing
}

\section{Conduct Material}

\author{
Xiaodan Liang ${ }^{1 \mathrm{a}}$, Na Lin ${ }^{2 \mathrm{~b}}$, Hanning Chen ${ }^{1 \mathrm{c}}$ and Wenxin $\mathrm{Liu}^{3 \mathrm{~d}}$ \\ ${ }^{1}$ Tianjin Polytechnic University, Tianjin, 300387, China \\ ${ }^{2}$ Beijing Shenzhou Aerospace Software Technology Co. Ltd., Beijing, China \\ ${ }^{3}$ Department of Electrical and Computer Engineering, Lehigh University, Bethlehem, USA. \\ alxdtjpu@163.com, ${ }^{b}$ linn@htrdc.com, ${ }^{c}$ perfect_chn@hotmail.com, ${ }^{\mathrm{d}}$ wliu@lehigh.edu
}

\begin{abstract}
Ink-jet printing circuit board has some advantage, such as non-contact manufacture, high manufacture accuracy, and low pollution and so on. In order to improve the and printing precision, the finite element technology is adopted to model the piezoelectric print heads, and a new bacteria foraging algorithm with a lifecycle strategy is proposed to optimize the parameters of driving waveforms for getting the desired droplet characteristics. Results of numerical simulation show such algorithm has a good performance. Additionally, the droplet jetting simulation results and measured results confirmed such method precisely gets the desired droplet characteristics.
\end{abstract}

Keywords. Finite Element Method (FEM), piezoelectric printhead, intelligent adjusting

\section{Introduction}

Ink-jet printing technology belongs to a kind of non-traditional machining process [1-2], which is also regarded as the increase-material manufacture, incremental, and rapid prototyping and so on. In recent 30 years, ink-jet printing technology is a collection of optical/mechanical/electrical, computer, numerical control and new materials in the integration of advanced manufacturing technology in global advanced manufacturing field. This technology deposits the powder, liquid, and flake on the substrate, which is different from the decimation method. This method greatly reduces the manufacturing complexity. Theoretically, as long as the design structure model on the computer, personnel can apply this technique to implement a fast manufacture without cutting tools, moulds and any complex design processes. The technique is particularly suitable for aerospace, weapons and equipment, in the field of biomedical, mold and other small batch and many asymmetric structure, surface and inner structure of empty parts (such as hollow aero engine blades, human bone restoration, conformal cooling channel) of rapid manufacturing, which conforms to the modern and the future development trend of manufacturing [3-4].

With the development of ink-jet printing technology, new problems also appear: (1) how to improve the efficiency of print? The efficiency of ink-jet printing technology manufacturing has a gap to the traditional manufacturing process (such as casting process) at mass production; (2) how to improve the stability of the printing? The stability of the ink jet printing technology is insufficient. In practice, it is difficult to guarantee the consistency of printing process, which causes printing product yield high enough; (3) how to improve the accuracy of print? Ink-jet printing precision greatly relies on 
the precision of the printhead. In terms of the present obtained industrial printhead, the adjustable range is very large, which has a lot of room to improve.

At present, in view of the above issues, in project, a kind of effective way is to optimize the choice of the working state of the printhead [5-8]: (1) improving print nozzle jet frequency and printing efficiency; (2) improving roundness of droplet ejected out of nozzle and enhancing the fault tolerance of printhead; (3) reducing the droplet volume ejected out of printhead and improving the print resolution. So it is need to control printhead to improve the print quality.

In this paper, we propose a new printhead intelligent adjusting method, which adopts the finite element method (FEM) to simulate the piezoelectric printhead jetting process. In order to improve the optimization efficiency, a new bacteria foraging algorithm with a lifecycle strategy is proposed to optimize the parameters of driving waveforms for getting the desired droplet characteristics.

The rest of this paper is organized as follows: In Section 2, the structure and working principle of piezoelectric printhead are introduced. The FEM-based intelligent adjustment system is described in Section 3. The bacteria foraging algorithm with a lifecycle strategy is proposed in Section 4. Section 5 demonstrates the simulation and experimental results. Finally, concluding remarks are collected in Section 6.

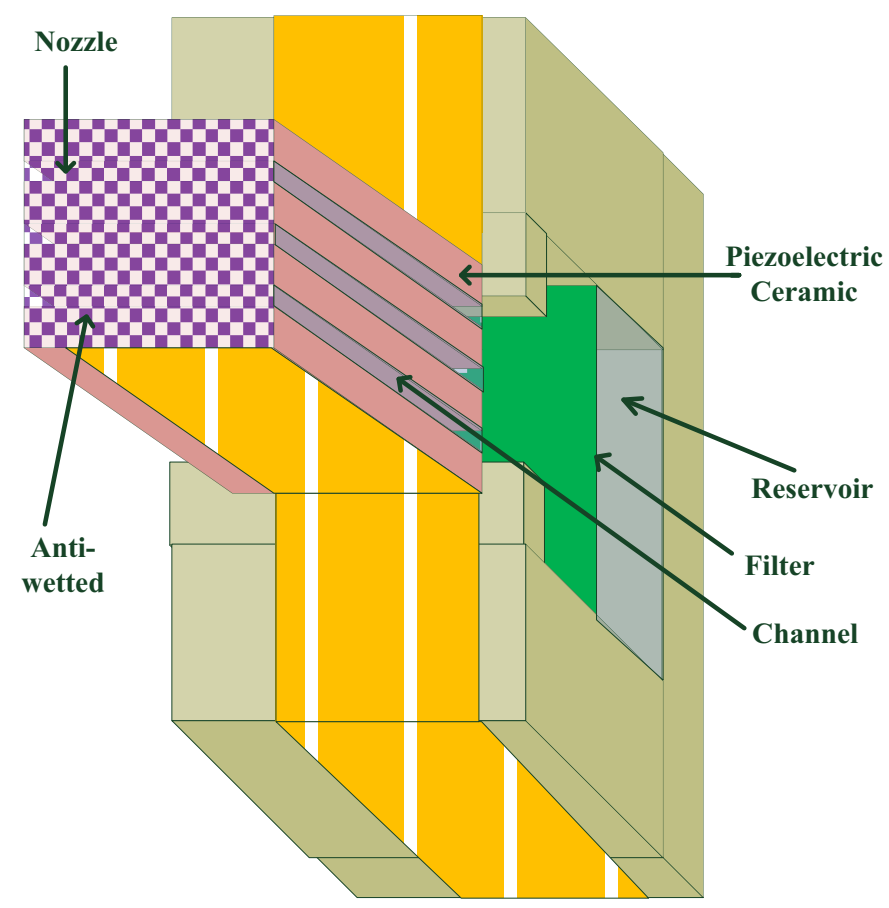

Fig. 1. Structure of Piezoelectric Printhead.

\section{Piezoelectric Printhead}

In this paper, the investigated printhead is KM series IJ Head of Konica. This is a typical piezoelectric (PZT) printhead. This kind of printhead has two arrays of nozzles in parallel. The structure of piezoelectric printhead is shown in Figure 1. Due to the need to spray printing ink mostly in high viscosity, it is necessary to heat the ink for decreasing ink viscosity. From Fig. 1, it is obvious that the nozzle has a reservoirwith a relatively large space; such design can effectiivly suppress the redundancy in ink-jet behavior after droplet jetting. The knitting filter can filter most of bubbles, particles pollution 
and large diameter particles to avoid them into ink channel, even to plug nozzle. Electric field on external electrode, making piezoelectric element deformation occurred. The change of the piezoelectric ceramic in ink channel generates pressure wave, and then pressure wave from the ink spreads to connect channel, through a narrow neck spreads to the spray hole, eventually forming droplets. Anti-wetted surface is to prevent the residual in the droplets on the surface of the nozzle clogging the nozzle.

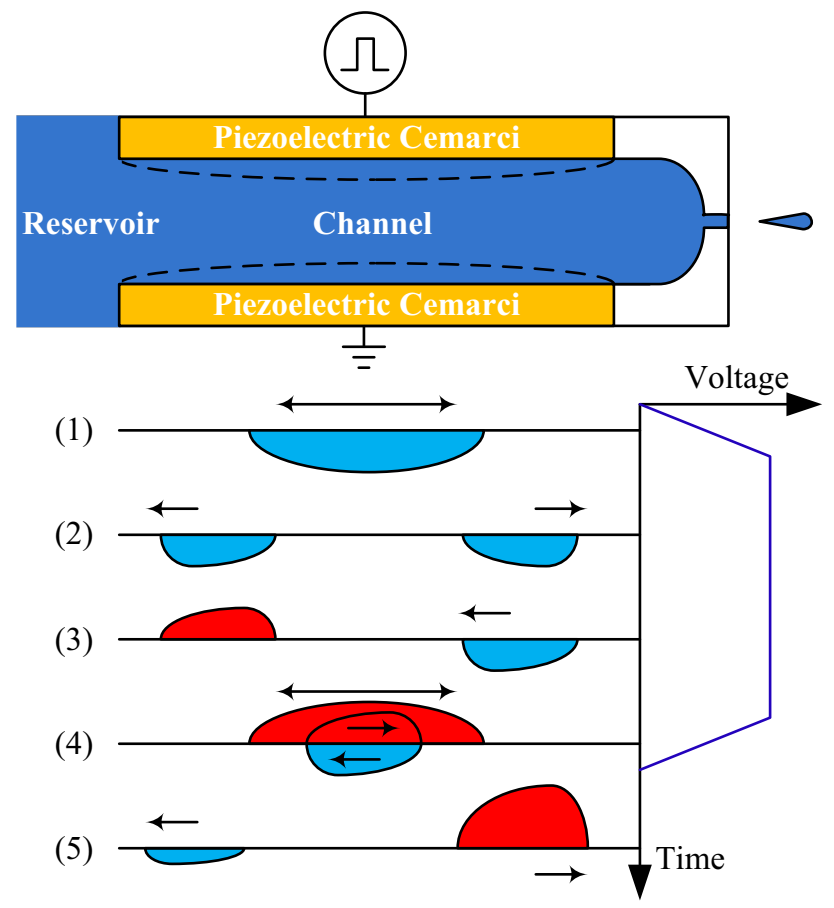

Fig. 2. Working principle of piezoelectric ink-jet printhead.

Figure 2 shows the cross-sectional schematic diagram of the ink channel. Ink channel length is greater than the diameter of the nozzle in the right hand side, and much smaller than the left side for the ink well geometry size. Figure 2 also shows a positive voltage applied to piezoelectric ceramics in trapezoidal wave when the pressure changes channel in the diagram.

\section{FEM-based Intelligent Adjustment System}

This research adopts the Finite Element Method (FEM) to simulate the working state of printheads. Finite element method is a kind of efficient and commonly used numerical method. Scientific computing, often need to solve all kinds of differential equation, and many general is hard to get the analytical solution of differential equation. Using the finite element method, the differential equation discretizes after programming with computer aided solution. At present, the widely used finite element analysis has been successfully applied to the fluid mechanics, electromagnetic mechanics, and structure mechanics calculation and so on. Using ANSYS and COMSOL finite element software for simulation, the preparatory design stage replaces the experimental testing for cost savings. 


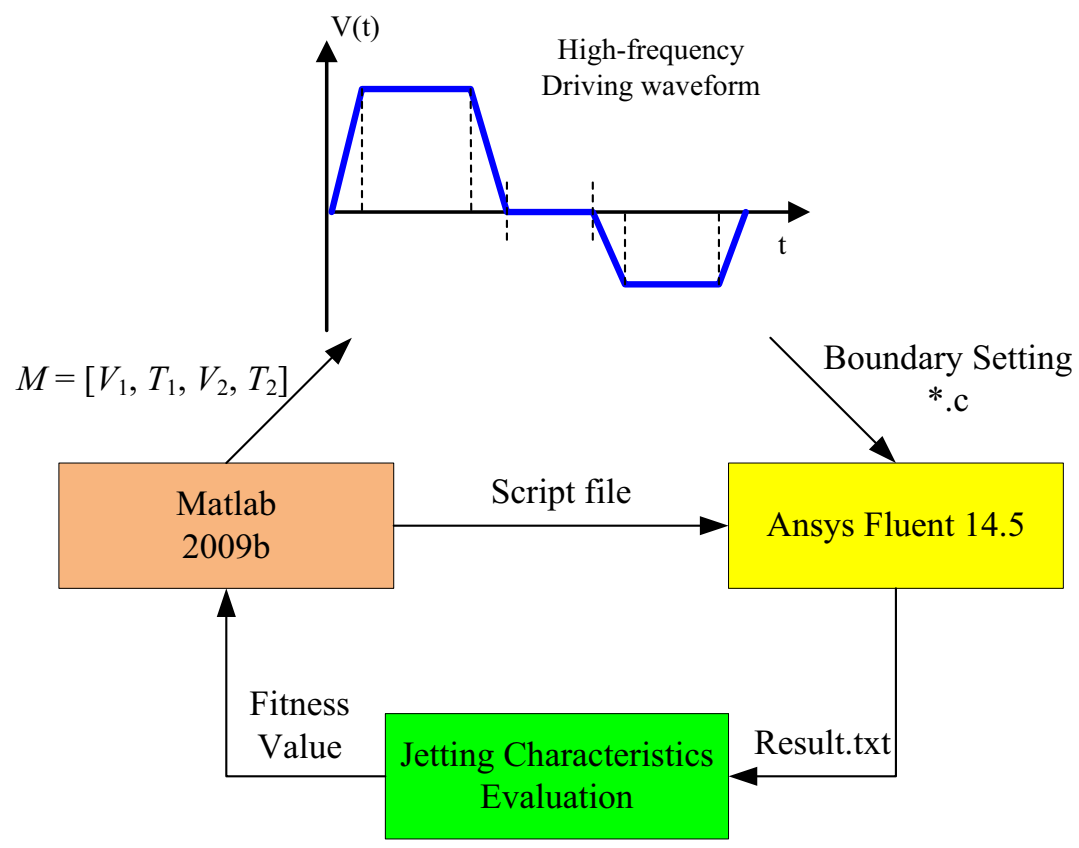

Fig.3. The simulation framework of jetting state.

This study sets a simulation framework of the printheand nozzle jetting state, as shown in Figure 3. Matlab script file generates the script file to drive FLUENT. The pressure generated by the intelligent optimization algorithm for FLUENT boundary configuration file is generated, too. The FLUENT software simulates the jetting process out of the nozzle according to the script files and configuration files, and generates the simulation results. According to preset evaluation standard, the fitness value is returned to the Matlab. According to the return of fitness value and the current waveform of driving parameter vector, intelligent algorithm generates the next generation of individuals, which drives the next cycle until the termination conditions meeting.

\section{Bacteria Foraging Algorithm with a Lifecycle Strategy}

The E. coli bacteria is one of the earliest bacteria which has been researched.Passino proposed the Bacterial ForagingOptimization (BFO) algorithm. The main mechanisms of BFO are illustrated as three steps: Chemotaxis, Reproduction and Eliminate-Dispersal. However, the original BFO has some shortages: (1) dispersal, reproduction, andelimination each happens; after a certain amount of chemotaxis operations. The appropriatetime andmethod for dispersal and reproduction are important. Otherwise, the stability of thepopulation may be destroyed. (2) The tumble angles in the chemotactic phase are generatedrandomly. As a result, the algorithm is more like a random searching algorithm except it willtry further in better directions. The bacteria swarm lacks interaction between individuals. Good information carried by those individuals in higher nutritional are as cannot be sharedwith and utilized by other bacteria. (3) The swim step length in the original BFO algorithm isa constant. In most cases, the bacterium will run one more step if the position is better than itslast position. If the swim step is large at the end stage (e.g., larger than the distance betweenits current position and the optimal point), it will skip the optimal point repeatedly. This will make the bacteria hard to converge to the optimal point.

To improve the optimization ability of BFO algorithms, for each bacterium, we will decide it to reproduce, die, or migrate by certainconditions in the bacteria's lifecycle. 
We define the nutrient updating formula as Eq. 1. Flast represents the fitness ofthe bacterium's last position (for a minimum problem, fitness is larger when the functionvalue is smaller). In initialization stage, nutrients of all bacteria are zero.

$$
N(i)= \begin{cases}N(i)+1 & \text { if }(F(i)>\text { Flast }) \\ N(i)-1 & \text { if }(F(i)<\text { Flast })\end{cases}
$$

In initialization stage, nutrients of all bacteria are zero. In the bacterium'schemotactic processes, if the new position is better than the last one, it is regarded that the bacterium will gain nutrient from the environment and the nutrient is added by one. Otherwise, it loses nutrient in the searching process and its nutrient is reduced by one.

\section{Results}

Lithium titanate solution (nanometer lithium titanate $35 \mathrm{~g}+$ alcohol $750 \mathrm{ml}+$ ethylene glycol $250 \mathrm{ml}$ ) is choosen as test ink, its physical properties as shown in Table 1.

Table 1. Physical characteristic of test ink.

\begin{tabular}{|c|c|c|c|}
\hline & Parameter & Unit & Value \\
\hline \multirow{3}{*}{ Ink } & Free electrical capacitance, $\sigma$ & $\mathrm{N} / \mathrm{m}$ & $45^{*} 10^{-3}$ \\
\cline { 2 - 4 } & Viscosity, $u$ & $\mathrm{Kg} /(\mathrm{m} * \mathrm{~s})$ & 15 \\
\cline { 2 - 4 } & Density, $\rho_{0}$ & $\mathrm{Kg} / \mathrm{m}^{3}$ & $1.05 * 10^{3}$ \\
\hline
\end{tabular}

At $50 \mathrm{~ms}$ after ejected droplet size, the speed of the front of droplet and the tail length as print nozzle are selected as the jet evaluationstates, and the evaluation function is as follows:

$$
F=m_{1} * \frac{\left|V_{\mathrm{S}}-V_{\mathrm{G}}\right|}{V_{\mathrm{G}}}+m_{2} * \frac{\left|S_{\mathrm{S}}-S_{\mathrm{G}}\right|}{V_{\mathrm{G}}}+m_{3} * \frac{L}{D_{\text {Nozzle }}}
$$

where $V_{G}$ is the desired droplet velocity, $V_{S}$ is the optimized droplet velocity, $S_{G}$ is the desired droplet volume, $S_{S}$ is the optimized droplet volume, $L$ is the tail length, $D_{\text {nozzle }}$ is the diameter of nozzle and $m_{1}, m_{2}$ and $m_{3}$ are the user-defined weights.

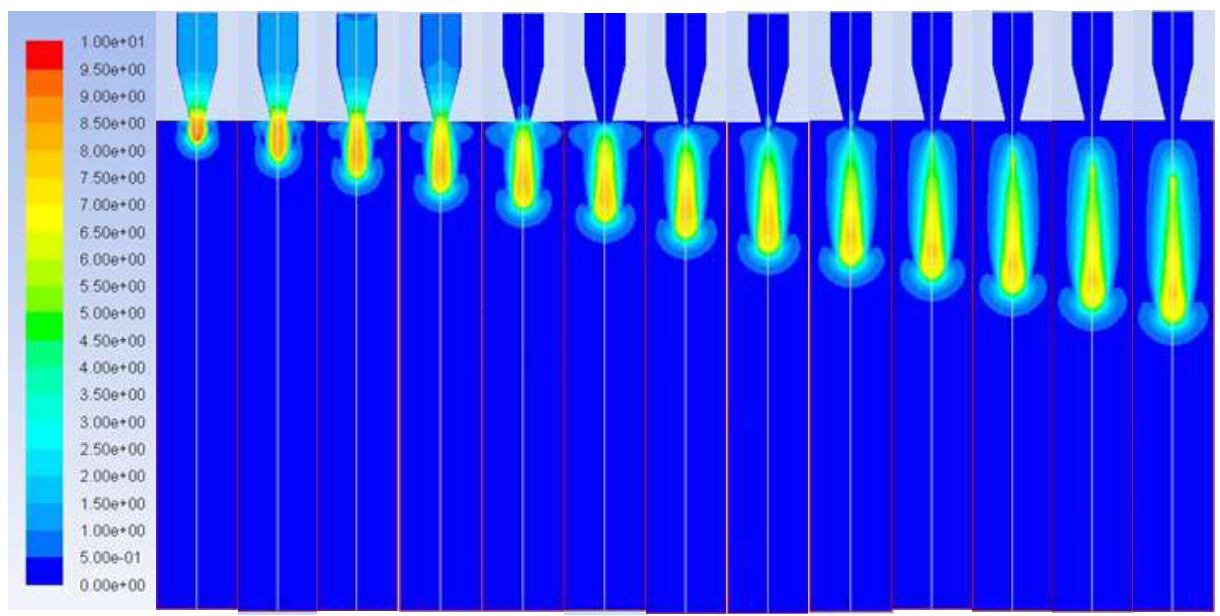

Fig. 4. Jetting characteristics optimized by the improved BFO 
Through the simulation results, as shown in Fig. 4, it is obvious that LBCFO algorithm simulation waveform parameters get expected droplet size and velocity, minimum error, the much excellent results. LBCFO algorithm is very suitable for optimizing the piezoelectric printhead for printing conduct material.

\section{Conclusion}

On account of the research about information communication mode and life period searching approach, we propose a new algorithm: Life-cycle Bacterial Colony Foraging Optimization. In order to verify the performance of the proposed new type of bacterial swarm foraging algorithm for complex engineering optimization problems, the experiments of inkjet printing system in intelligent manufacturing field have been tested. The mode of printhead is structured in ANSYS software. The proposed algorithm got a small error towards target droplet velocity and volume.

\section{Acknowledgement}

This research is partially supported by National Natural Science Foundation of China (Grand Nos. 51575158, and 61305082), and a self-planned task of CAS Key Laboratory of Networked Control System (Grand No. WLHKZ2015001).

\section{References}

1. E. Macdonald, R. Salas, D. Espalin, M. Perez, E. Aguilera, D. Muse and R.B. Wicker, 3D Printing for the Rapid Prototyping of Structural Electronics, IEEE Access, 2 (2014), p. 234-242.

2. Information on https://en.wikipedia.org/wiki/3D printing

3. G.K. Jan, J. Patrickand D.Y. Shin, in: Inkjet-based Micro manufacturing, Wiley (2012).

4. Information on https://en.wikipedia.org/wiki/Chuck_Hull

5. J. Sun, J, R Yang, K K Tan, J Y H Fuh and Y S Wong, Performance characterization of drop-on-demand micro-dispensing system with multi-printheads, Microsystem Technologies, , Vol. 16, no. 12 (2010), p. $2087-2097$

6. A.K. Amol, X. Bombois, G. Scorletti, R. Babuška, S. Koekebakker, and W. Zeeuw, A Waveform Design Method for a PiezoInkjetPrinthead Based on Robust Feedforward Control, Journal of Microelectromechanical Systems, 21, no. 6) (2012), p. 1365-1374

7. A.L. Yarin, Drop Impact Dynamics: Splashing,Spreading, Receding, Bouncing, Annu. Rev. Fluid Mech, 38 (2006), p.159-92

8. B. Derby, Inkjet printing ceramics: From drops to solid, Journal of the European Ceramic Society, 31 (2010), p. $2543-2550$. 